\title{
Prefrontal cortical and striatal transcriptional responses to the reinforcing effect of repeated methylphenidate treatment in the spontaneously hypertensive rat, animal model of attention-deficit/ hyperactivity disorder (ADHD)
}

Ike dela Peña', Hee Jin Kim', Aeree Sohn', Bung-Nyun Kim², Doug Hyun Han³, Jong Hoon Ryu4, Chan Young Shin ${ }^{5}$, Minsoo Noh $^{6 *}$ and Jae Hoon Cheong ${ }^{1 *}$

\begin{abstract}
Background: Methylphenidate is the most commonly used stimulant drug for the treatment of attention-deficit/ hyperactivity disorder (ADHD). Research has found that methylphenidate is a "reinforcer" and that individuals with ADHD also abuse this medication. Nevertheless, the molecular consequences of long-term recreational methylphenidate use or abuse in individuals with ADHD are not yet fully known.

Methods: Spontaneously hypertensive rats (SHR), the most validated and widely used ADHD animal model, were pretreated with methylphenidate (5 mg/kg, i.p.) during their adolescence (post-natal day [PND] 42-48) and tested for subsequent methylphenidate-induced conditioned place preference (CPP) and self-administration. Thereafter, the differentially expressed genes in the prefrontal cortex (PFC) and striatum of representative methylphenidate-treated SHRS, which showed CPP to and self-administration of methylphenidate, were analyzed.
\end{abstract}

Results: Genome-wide transcriptome profiling analyses revealed 30 differentially expressed genes in the PFC, which include transcripts involved in apoptosis (e.g. S100a9, Angpt/4, Nfkbia), transcription (Cebpb, Per3), and neuronal plasticity (Homer1, Jam2, Asap 1). In contrast, 306 genes were differentially expressed in the striatum and among them, 252 were downregulated. The main functional categories overrepresented among the downregulated genes include those involved in cell adhesion (e.g. Pcdh10, Ctbbd1, Itgb6), positive regulation of apoptosis (Perp, Taf1, Api5), (Notch3, Nsbp1, Sik1), mitochondrion organization (Prps18c, Letm1, Uacrc2), and ubiquitin-mediated proteolysis (Nedd4, Usp27x, Ube2d2).

Conclusion: Together, these changes indicate methylphenidate-induced neurotoxicity, altered synaptic and neuronal plasticity, energy metabolism and ubiquitin-dependent protein degradation in the brains of methylphenidate-treated SHRs, which showed methylphenidate CPP and self-administration. In addition, these findings may also reflect cognitive impairment associated with chronic methylphenidate use as demonstrated in preclinical studies. Future studies are warranted to determine the clinical significance of the present findings with regard to long-term recreational methylphenidate use or abuse in individuals with ADHD.

Keywords: Methylphenidate, ADHD, Gene expression, Addiction

\footnotetext{
* Correspondence: minsoonoh@snu.ac.kr; cheongjh@syu.ac.kr

${ }^{6}$ Natural Products Research Institute, College of Pharmacy, Seoul National University, 1 Gwanak-ro, Gwanak-gu, Seoul 151-742, Korea

'Uimyung Research Institute for Neuroscience, Sahmyook University, 26-21

Kongreung-2-dong, Hwarangro- 815 Nowon-gu, Seoul 139-742, Korea

Full list of author information is available at the end of the article
} 


\section{Background}

Central nervous system (CNS) stimulants (e.g. methylphenidate and amphetamines) are recommended as first line medications for attention-deficit/hyperactivity disorder (ADHD [1]. Among the psychostimulants, methylphenidate is the most commonly prescribed [1]. Methylphenidate blocks the dopamine transporter (DAT), the key mechanism responsible for the removal of extracellular dopamine (DA), thereby elevating extracellular DA levels in various limbic, striatal, cortical, cerebellar terminal fields and increasing DA signaling and duration of DA response [2]. Neuroimaging studies showed that therapeutic doses of methylphenidate increased DA levels in the striatum and nucleus accumbens (NAc), a mechanism purportedly thought to explain methylphenidate-induced improvement of ADHD symptoms [3,4]. However, in light of the observation that the effects of methylphenidate are similar to those exerted by other drugs affecting the CNS (e.g. addictive drugs such as cocaine or methamphetamine), the abuse potential of methylphenidate has been suggested [5].

The repeated use of stimulants can elicit adverse effects in behavior and induce drug-related behaviors such as sensitization, tolerance or dependence [6-8]. Therefore, the propensity of long-term methylphenidate treatment to induce drug addiction or dependence in individuals with ADHD has also been implied. A number of studies have investigated effects of methylphenidate treatment on subsequent abuse of other addictive drugs [9-13]. Unfortunately, however, only a few studies have sought to determine whether the repeated treatment of methylphenidate will result in the abuse of the drug itself. Furthermore, the long-term effects of repeated methylphenidate use in behavior and gene expression remain far from understood [14]. In particular, there is a dearth of information on the potential neuronal correlates of long-term recreational methylphenidate use or abuse in individuals with ADHD $[8,14]$.

Preclinical studies in animal models have provided an avenue for identifying the potential molecular neuropathobiology of drug-induced neuroadaptations [15]. Moreover, the use of microarray expression profiling has aided us in capturing neuroadaptations involving complex changes in gene expression that may underlie the development of drug addiction $[15,16]$. The early microarray studies have shown that repeated methylphenidate treatment even at a clinically relevant dose produced changes in gene regulation in cortical and striatal neurons which are similar to those of cocaine and amphetamine, indicating addiction liability of the drug [14]. While identification of these gene sets represents a significant step, it is likely that there are other changes that have not been identified, especially those that result from the behavioral or cognitive processes associated with methylphenidate use, in addition to the direct pharmacological effects of the drug [17-19]. Indeed, by integrating methylphenidateinduced gene expression changes with outcomes in "appropriate" animal models of drug addiction (e.g. conditioned place preference $[\mathrm{CPP}]$ tests and drug self-administration), we identified a subset of neuronal development genes, which may also mediate the reinforcing effect of methylphenidate [19]. We suggested significant clinical implications of these findings on the abuse of methylphenidate among "healthy" individuals, as our studies [19] were conducted in Wistar rats, strain used to represent the "normal" heterogeneous population. However, the previous findings could not be generalized to individuals with ADHD who are also abusing methylphenidate, as experiments were not conducted in "appropriate" ADHD animal models.

To address this issue at the preclinical level and to shed some light on the potential molecular consequences of long-term recreational methylphenidate use or abuse in individuals with ADHD, we analyzed the differentially expressed genes (DEGs) in the brain of methylphenidatepretreated Spontaneously Hypertensive rats (SHRs), which showed CPP to and self-administration of methylphenidate. The SHR, relative to the normotensive Wistar Kyoto rat strain, exhibits good face, construct and predictive validity mimicking the behavioral characteristics observed in ADHD, and is considered as the most widely used and validated animal model for this disorder [20,21]. Abnormalities both in the genetic and neurotransmitter functions, such as those seen in ADHD were also observed in the SHR [22,23]. Moreover, earlier studies have found increased reactivity of the SHR to stimulants, opioids, alcohol and other addictive drugs in comparison with other strains, indicating that the SHR, in general, may be used to investigate the relationship between ADHD and drug addiction [24]. Most studies on the molecular effects of stimulants have focused on gene regulation in DA target areas such as the striatum $[25,26]$. In this regard, genome-wide transcriptional analyses were performed in the striatum because this brain region controls reward sensitivity, motor function and habit learning [27]. Furthermore, prefrontal cortices (PFC) were also included in these analyses because they include regions involved in processes relevant to drug addiction such as compulsive drug taking, and maintenance of behavioral sensitization [28,29].

\section{Methods}

\section{Animals and drug treatment}

All experiments were performed in accordance with the Principles of Laboratory Animal Care (NIH) and the Animal Care and Use Guidelines of Sahmyook University, Korea. Male SHRs were purchased from Orient Co. Ltd., a branch of Charles River Laboratories (Seoul, Korea) and housed in an environmentally-controlled animal room (temperature $\left[22 \pm 2^{\circ} \mathrm{C}\right.$ ] and humidity [ $55 \pm 5 \%$ ], 
$12 \mathrm{~h} / 12 \mathrm{~h}$ light/dark [6 AM-6 PM] cycle) in groups during drug treatment and conditioned place preference (CPP) experiments, and individually thereafter during self-administration tests. After a 1 week of acclimatization, adolescent (6 weeks old) SHRs were given saline [ $(1 \mathrm{mg} / \mathrm{kg}$, intraperitoneal (i.p.), cohort 1] or racemic $d l$-methylphenidate (obtained from Hwanin Pharmaceutical Co. Korea), twice daily [at 9 AM and 9 PM], for 7 days (cohort 2) at a dose (5 $\mathrm{mg} / \mathrm{kg}$, i.p.) that produced a CPP response to the drug $[19,30]$, and evaluated for subsequent methylphenidate CPP and self-administration. Another group of SHRs (Control) was pretreated with saline and given saline only during all behavioral assays. Methylphenidate pretreatment was started during adolescence, in view of the findings that treatment initiation during adolescence increased risk of polydrug use [31,32], and in light of the observation that methylphenidate pre-exposure during adolescence increases behavioral response of rats to the reinforcing effects of addictive drugs [for review see [8]]. Furthermore, since our goal was to "model" recreational use of methylphenidate in individuals with ADHD, SHRs were pretreated with methylphenidate at a dose beyond the clinically-relevant range $(0.5-2 \mathrm{mg} / \mathrm{kg}$,i.p.), and administered with methylphenidate intraperitoneally, a method which is twice as potent as oral methylphenidate administration in increasing extracellular DA levels [33]. Of note, while drug treatment was conducted during both light and dark phases of the light/dark cycle, all behavioral tests were conducted during the light portion of the day only (between 9 AM and 5 PM).

\section{Conditioned place preference (CPP) tests}

CPP tests, conducted a day after the final drug or saline administration, were performed following the methods described previously [30,34]. Tests were performed in a two-compartment CPP apparatus described in previous studies [30,34]. Animal movement and behavior were video-recorded and analyzed using the Ethovision (Noldus, Netherlands) system. The CCP tests consisted of three phases: habituation and preconditioning (3 days), conditioning (6 days) and post-conditioning (1 day). After two days of habituation sessions, initial preference of rats for one compartment of the CPP box was measured for 15 minutes. The conditioning phase followed (6 days) during which cohort 1 and 2 rats were injected with methylphenidate $(5 \mathrm{mg} / \mathrm{kg}$, i.p.) and confined to their initially non-preferred side for $30 \mathrm{~min}$ [30,34], and given saline $(1 \mathrm{mg} / \mathrm{kg}$, i.p.) and confined to their preferred side on alternate days. Meanwhile, rats of the control group received saline only during the conditioning days. The postconditioning phase followed the next day and the time spent by each rat in each compartment of the CPP apparatus was recorded for $15 \mathrm{~min}$. After the post-conditioning phase, the shift in place preference was determined. CPP data were expressed as the difference in time spent in the drug- or saline-paired compartment during the post and preconditioning phases. The results were presented as the means \pm SEM and statistical analysis was performed using unpaired $t$-tests. A $P$ value of $<0.05$ was regarded as significant.

\section{Self-administration tests}

The rats that showed robust CPP to methylphenidate were used for further tests ( $n=7-9$ rats per group) [19]. Six rats from the control group were also used in this study. Rats were trained to press a lever for a sucrose pellet reward and when lever-pressing behaviors were stabilized, they were implanted with silastic catheters in the right jugular vein following methods described in our previous study [34]. Following recovery from surgery, rats underwent 6 days of 2-h methylphenidate or saline (control group) self-administration under the FR1 schedule. The methods of the self-administration tests are outlined in our previous study [34]. Each active lever press resulted in an infusion of $0.1 \mathrm{ml}$ saline $(0.9 \% \mathrm{NaCl})$, or methylphenidate $(0.25 \mathrm{mg} /$ $0.1 \mathrm{ml}$ infusion) [34] for cohort 1 or 2 groups, respectively. The number of saline or methylphenidate infusions administered by rats over the 6 days of self-administration was recorded. The results are presented as the means \pm SEM and two-way ANOVA was used for data comparison. A $P$ value of $<0.05$ was regarded as significant. All statistical analyses (CPP and self-administration tests) were conducted using GraphPad Prism Version 5 software (San Diego, CA, USA).

\section{Tissue collection and RNA preparation}

One day after the final self-administration session, we removed the brains of 3 rats from cohort 2 (i.e., those which showed the most robust self-administration) as well as 3 rats from the control group for microarray analyses. We used this time point to eliminate confounding results induced by the direct effects of the drug [19]. After decapitation, the brains were rapidly removed and placed in ice-cold saline. The striatum (rostral part of the caudate, putamen and the NAc) and the prefrontal cortex were removed and immediately frozen at $-70^{\circ} \mathrm{C}$. Total RNA was isolated using Trizol reagent (Invitrogen, Carlsbad, CA, USA) according to the manufacturer's instructions. The RNA was further purified using the RNEasy mini kit (Qiagen Inc.). The total RNA concentration was determined using NanoDrop ND-1000 Spectrometer (NanoDrop Technologies Inc., Montchanin, DE, USA). The RNA integrity was assessed using a BioAnalyzer 2100 (Agilent Technologies, Santa Clara, CA, USA).

\section{Oligonucleotide microarray analyses}

Affymetrix GeneChip microarrays were prepared, hybridized and scanned by the local authorized Affymetrix service 
provider (DNA Link, Inc., Seoul, South Korea). The RNA was converted to cDNA and transcribed into cRNA in the presence of biotinylated ribonucleotides, according to standard Affymetrix protocols (Affymetrix 'Expression Analysis Technical Manual', \#701021 Rev. 5). Biotin-labeled aRNA were transcribed in vitro following the Affymetrix GeneChip manufacturer's protocol. Hybridization was performed using Affymetrix GeneChip Rat Genome 2302.0 oligonucleotide arrays (Affymetrix, Santa Clara, CA, USA). The hybridized probe array was stained and washed with a GeneChip hybridization, wash and stain kit using the Fluidics Station 450 (Affymetrix). The stained GeneChip probe array was scanned with a GeneChip Scanner $3000+7 G$ (Affymetrix). The signal intensity of the gene expression level was calculated using Expression Console Software, Version 1.1 (Affymetrix) based on the MAS 5.0 algorithm. The procedure used to select DEGs was as follows: (i) selection of "present" Affymetrix probe sets for 3 baseline or treated tissue samples; (ii) selection of "up-regulated" and "down-regulated" Affymetrix probe sets with comparison signal sample/control ratios $>1.65$ and $<0.6$, respectively, and whose values corresponded to average ratio values at the $2.5 \%$ tails of three microarray histograms; and (iii) selection of Affymetrix probe sets with simultaneously significant $P$ values (threshold, 0.05 ) in the Wilcoxon rank test when compared with vehicle-treated samples.

\section{Gene ontology analysis}

The Database for Annotation, Visualization and Integrated Discovery (DAVID) v6.7 functional clustering tool was used to identify over-represented ontologic groups among the gene expression profiles and to group DEGs into functional categories [35]. Gene Ontology Biological Process was selected as the functional annotation category for this analysis.

\section{Validation of microarray data by qRT-PCR}

qRT-PCR was performed to validate microarray data on selected genes. Briefly, 1 microgram of total RNA, obtained from samples used for microarrays, was reverse-transcribed into cDNA using SuperScript TM reverse transcriptase (Invitrogen), and aliquots were stored at $-20^{\circ} \mathrm{C}$. The gene identification number for the TaqMan probes (Applied Biosystems) used in the qRT-PCR analyses are shown in Table 1. Rat GAPDH (Applied Biosystems) was also amplified to normalize the variations in cDNA quantities from different samples. The qRT-PCR reactions were performed in triplicates, and the qRT-PCR data were represented as $\mathrm{Ct}$ values, where $\mathrm{Ct}$ is defined as the threshold cycle. The relative differences in gene expressions were quantified using equations from a previously developed mathematical model [36]. The results are presented as the means \pm SEM, and statistical analysis was performed using
Table 1 Gene identification numbers for Taqman probes used in the qRT-PCR analysis

\begin{tabular}{ll}
\hline Gene symbol & Probe set ID \\
\hline Angptl4 & Rn01528817_m1 \\
Cttnd1 & Rn01486371_m1 \\
Gapdh & Rn01775763_g1 \\
Homer1 & Rn00581785_m1 \\
Nedd4 & Rn01530544_m1 \\
Pcdh10 & Rn01294983_m1 \\
Perp & Rn01440467_m1 \\
S100a9 & Rn00585879_m1 \\
Uacrc2 & Rn01506370_m1 \\
\hline
\end{tabular}

one-way ANOVA followed by Bonferroni's post-test. A $P$ value of $<0.05$ was regarded as significant.

\section{Results}

Reinforcing effect of repeated methylphenidate treatment in SHRs

Figure 1 shows that methylphenidate-CPP was expressed by methylphenidate-pretreated SHRs conditioned with

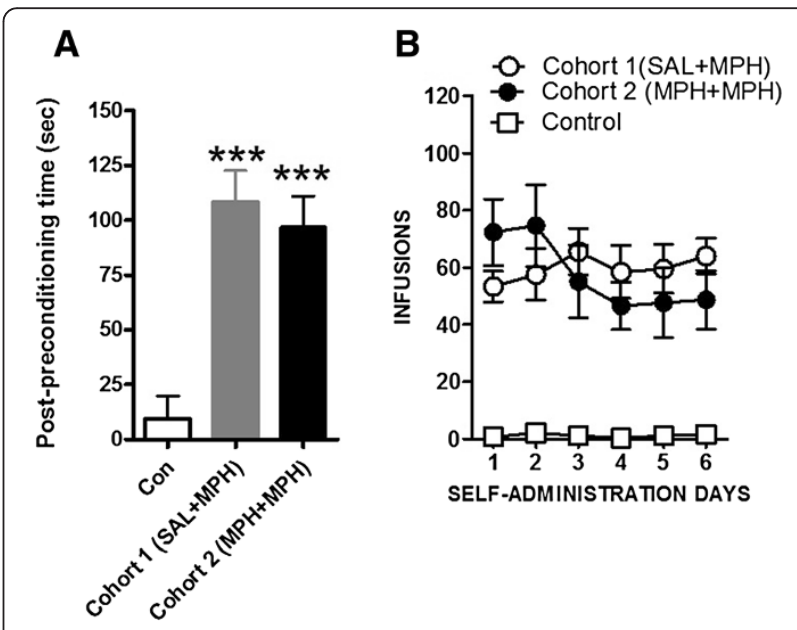

Figure 1 Reinforcing effects of repeated methylphenidate treatment in spontaneously hypertensive rats (SHRs).

(A) Methylphenidate (MPH) CPP in cohort 1 (saline-pretreated SHR conditioned with methylphenidate, SAL + MPH) and cohort 2 (methylphenidate-pretreated SHR conditioned methylphenidate, $\mathrm{MPH}+\mathrm{MPH}$ ) rats. During the conditioning phase of the CPP test, the rats in both cohorts were administered $5 \mathrm{mg} / \mathrm{kg}$ dose of $\mathrm{MPH}$. The control group received saline only. Each bar represents the mean \pm S.E.M. of the difference in the time spent in the drug- or saline(for control group) paired side during the post- and preconditioning phases. ${ }^{* *} P<0.001$, significantly different from the saline-treated groups. $n=10-12$ rats per group. (B) MPH self-administration shown by saline-treated (SAL + MPH) and methylphenidate-pretreated $(\mathrm{MPH}+\mathrm{MPH}) \mathrm{SHR}$. The mean number of methylphenidate infusions obtained by rats over the 6 days of methylphenidate self-administration is shown. Each symbol represents the mean \pm S.E.M. $n=7-9$ animals per group. 
methylphenidate (cohort 2) $[t(20)=4.17, P<0.001]$ (Figure 1). Saline-pretreated SHRs conditioned with the drug (cohort 1) also showed CPP to methylphenidate $([t(18)=5.58, P<0.001]$ and the average CPP scores between cohort 1 and 2 rats did not vary significantly $[t(20)=0.95, P>0.05]$. Out of the 12 animals in cohort 2, we selected 9 rats with the highest CPP scores for further self-administration tests. Seven rats from cohort 1 (i.e., rats with the highest CPP scores) and 7 rats from the control group were also chosen for further selfadministration studies. Figure 1B shows the number of methylphenidate infusions obtained by SHRs. Selfadministration tests showed reinforcing effects of methylphenidate in cohort $2[F(1,84)=106.0, P<0.001]$ as well as in cohort 1 SHRs $[F(1,72)=333.4, P<0.001]$ and two-way ANOVA revealed no significant difference in the rate of responding for methylphenidate infusions between the two groups $[F(1,84)=0.13, P>0.05]$. In summary, the findings from both CPP and self-administration studies indicate reinforcing effect of methylphenidate in drug-pretreated SHRs.

\section{Transcriptional responses to the reinforcing effect of repeated methylphenidate treatment in the prefrontal cortex and striatum of the SHR}

With regard to the objective of this study, we analyzed prefrontal cortical and striatal gene expression changes in methylphenidate-pretreated SHRs, which showed CPP to and self-administration of methylphenidate. Genome-wide transcriptional profiling showed that 30 transcripts were differentially regulated in the PFC (Table 2), while 306 genes were differentially expressed in the striatum of cohort 2 SHRs vs. controls (see Additional file 1: Tables S1A and $1 \mathrm{~B}$ for complete list of differentially expressed genes in the striatum). Interestingly, a majority $(82 \%)$ of the DEGs in the striatum were downregulated genes (Additional file 2: Table S2). The differentially expressed genes (DEGs) in the PFC belong to functional categories such as regulation of apoptosis (Nfkbia, S100a9, Angptl4), transcription (Cebpb, Dbp, Per3) and cell migration (Abcc9, Ctgf) (Table 2). The main functional categories overrepresented among the upregulated genes in the striatum include those involved in synaptic transmission (Grm8, Shank1, Camk2n1, Pja2), negative regulation of apoptosis (Sap30bp, Apoe, Nfkbia), transcription ( Dbp, Klf2, Neurod2), and others (Additional file 2: Table S2). Categories overrepresented among the downregulated genes are those associated with cell adhesion (Pcdh10, Ctbbd1, Itgb6), positive regulation of apoptosis (Perp, Taf1, Api5), transcription (Notch3, Nsbp1, Sik1), mitochondrion organization (Prps18c, Letm1, Uqcrc2), ubiquitin-mediated proteolysis (Nedd4, Usp27x, Ube2d2) and others (Additional file 2: Table S2).
qRT-PCR validations of selected differentially expressed genes qRT-PCR was used to confirm a subset of differentially expressed genes identified in the microarray studies. The genes selected for qRT-PCR confirmation were those representing the major functional gene families among the downregulated genes. The expression patterns of Pcdh10 and Ctnnd1, genes associated with cell adhesion were validated through qRT-PCR (Figure 2). We also confirmed microarray results with Perp, Nedd4 and Uqcrc2, genes associated with apoptosis, ubiquitin-mediated proteolysis, and mitochondria organization, respectively (Figure 2). Furthermore, we also found a correlation between expression patterns predicted by microarrays and those determined by qRT-PCR analyses for S100a9, Angptl4 and Homer1, DEGs in the PFC of cohort 2 SHRs. These findings strengthen the reliability of our microarray results.

\section{Discussion}

To model recreational methylphenidate use in individuals with ADHD, we subjected SHRs to repeated methylphenidate treatment (7 days, twice daily) at a dose (5 $\mathrm{mg} / \mathrm{kg}$, i.p.) beyond the clinically-relevant range (0.5-2 $\mathrm{mg} / \mathrm{kg}$, i.p.), and tested them for subsequent methylphenidate CPP and drug self-administration. In order to shed light on the potential molecular consequences of long-term recreational methylphenidate use or abuse in individuals with ADHD, we analyzed differentially expressed genes (DEGs) in the PFC and striatum of methylphenidate-pretreated SHRs, which showed methylphenidate reinforcement (i.e. CPP to and self-administration of methylphenidate). The findings from behavioral studies revealed methylphenidate CPP and acquisition of methylphenidate self-administration in methylphenidate-pretreated SHRs. Genome-wide analyses in the PFC and striatum of these rats showed that methylphenidate alters the expression of a number of genes involved in many functional systems. We discuss herein some of these important DEGs whose functions have already been characterized, and hence a role in methylphenidate reinforcement in SHRs can be extrapolated.

Previous studies showed that methylphenidate pretreatment at the $5 \mathrm{mg} / \mathrm{kg}$ dosage (i.p.) enhanced psychomotor response to cocaine in other rat strains (e.g. SpragueDawley rats and Wistar rats) [10]. Moreover, pretreatment at various dosages of methylphenidate also increased vulnerability to cocaine as well as to other addictive drugs $[8,10,11]$. We have previously reported enhancement of CPP response to methylphenidate in methylphenidatepretreated (5 mg/kg, ip., 7 days, twice daily) Wistar rats [19] indicating the occurrence of behavioral sensitization, a phenomenon associated with enhanced behavioral response to repeated treatment of addictive drugs [37]. Druginduced sensitization has been thought to underlie certain aspects of addiction; in particular, the neuroadaptations that 
Table 2 Differentially expressed genes in the prefrontal cortex (PFC) of cohort 2 SHRs relative to controls

\begin{tabular}{|c|c|c|c|}
\hline \multicolumn{4}{|c|}{ A. Upregulated genes } \\
\hline Probe set ID & Gene title & Gene symbol & $\begin{array}{l}\text { Mean fold } \\
\text { change }\end{array}$ \\
\hline & Regulation of apoptosis & & \\
\hline 1388924_at & Angiopoietin-like 4 & Angpt/4 & 1.78 \\
\hline 1389538_at & Nuclear factor of kappa light polypeptide gene enhancer in B-cells inhibitor, alpha & Nfkbia & 1.66 \\
\hline 1387125_at & S100 calcium binding protein A9 & S100a9 & 4.37 \\
\hline 1373302_at & Alkaline ceramidase 2 & Acer2 & 2.27 \\
\hline \multirow[t]{2}{*}{ 1391791_at } & Alkaline ceramidase 2 & Acer2 & 1.87 \\
\hline & Transcription & & \\
\hline 1387087_at & CCAAT/enhancer binding protein (C/EBP), beta & Cebpb & 1.78 \\
\hline 1387874_at & D site of albumin promoter (albumin D-box) binding protein & Dbp & 2.18 \\
\hline \multirow[t]{2}{*}{ 1378745_at } & Period homolog 3 (Drosophila) & Per3 & 1.68 \\
\hline & Protein complex assembly & & \\
\hline 1389234_at & Von Willebrand factor & $V w f$ & 2.17 \\
\hline 1367553_x_at & Hemoglobin, beta /// beta globin minor gene /// beta-globin & $\begin{array}{l}\text { Hbb /// LOC100134871 } \\
\text { /// LOC689064 }\end{array}$ & 2.82 \\
\hline 1370239_at & Hemoglobin alpha, adult chain 2 /// hemoglobin alpha 2 chain & Hba-a2 /// LOC360504 & 1.86 \\
\hline \multirow[t]{2}{*}{ 1370240_x_at } & Hemoglobin alpha, adult chain 2 /// hemoglobin alpha 2 chain & Hba-a2 /// LOC360504 & 1.86 \\
\hline & Cell migration & & \\
\hline 1398265_at & ATP-binding cassette, subfamily C (CFTR/MRP), member 9 & Abcc9 & 1.81 \\
\hline \multirow[t]{2}{*}{ 1367631_at } & Connective tissue growth factor & Ctgf & 1.68 \\
\hline & Miscellaneous & & \\
\hline 1371237_a_at & Metallothionein 1a /// transthyretin & Mt1a /// Ttr & 1.69 \\
\hline 1371447_at & Placenta-specific 8 & Plac8 & 1.80 \\
\hline 1384969_at & Collagen, type XXIV, alpha 1 & Col24a1 & 1.70 \\
\hline 1387658_at & Eukaryotic elongation factor-2 kinase & Eef2k & 1.69 \\
\hline 1379766_at & - & - & 1.66 \\
\hline 1381178_at & - & - & 2.15 \\
\hline 1386145_at & - & - & 2.23 \\
\hline 1389250_at & - & - & 1.71 \\
\hline \multicolumn{4}{|c|}{ B. Downregulated genes } \\
\hline 1370454_at & Homer homolog 1 (Drosophila) & Homer1 & 0.37 \\
\hline 1370997_at & Homer homolog 1 (Drosophila) & Homer1 & 0.46 \\
\hline 1380545_at & ArfGAP with $\mathrm{SH} 3$ domain, ankyrin repeat and PH domain 1 & Asap 1 & 0.58 \\
\hline 1393324_at & Junctional adhesion molecule 2 & Jam2 & 0.55 \\
\hline 1393451_at & Centromere protein $\mathrm{N}$ & Cenpn & 0.54 \\
\hline 1395309_at & - & - & 0.60 \\
\hline 1378507_at & - & - & 0.58 \\
\hline 1384410_at & - & - & 0.59 \\
\hline
\end{tabular}

accompany this phenomenon have been suggested to mediate the maintenance and reinstatement of addiction [37]. We observed in this study that methylphenidate pretreatment produced reinforcing effects in SHRs, however, we also found comparable CPP scores and similar rates of methylphenidate self-administration in both saline- and methylphenidate-pretreated rats (cohort 1 and 2 rats, respectively). Investigating the mechanism that underlies differential behavioral responses of strains to the reinforcing effects of repeated methylphenidate treatment is beyond 
the scope of this study [for review, however, see [38]]. Nevertheless, the present and the previous findings [19] appear to simulate the reported similarity in the rates of drug abuse or dependence to psychoactive substances in both ADHD and non-ADHD controls [39].

In general, our microarray studies revealed transcriptional changes associated with pharmacological effects of the drug or consequential to effects of chronic methylphenidate exposure (e.g. genes related with apoptosis, inflammation, etc.), although these analyses also found some DEGs whose expression may have been changed in response to the cognitive or behavioral processes associated with drug use (e.g. genes involved in neuronal and/or synaptic plasticity as well as in neuronal development) [[17,19], for review see [18]]. In the PFC, methylphenidate differentially altered the expression of genes involved in apoptosis (e.g. S100a9, Angptl4, Nfkbia), transcription (Cebpb,Per3), and neuronal plasticity (Homer1, Jam2, Asap1). qRT-PCR validated differential expression patterns of S100a9, a gene that encodes S100a9, which together with S100a8 exerts broad apoptotic activities [40]. Psychostimulants, including methylphenidate [41], activate the production of superoxides [42], inflammatory cells (astrocytes and microglia), and they in turn have been shown to release numerous pro-inflammatory factors and cytokines [43]. Moreover, psychostimulant-induced increases in synaptic and cytosolic DA levels have been shown to be neurotoxic due to DA-induced production of reactive oxygen species (ROS), reactive nitrogen species, hydrogen peroxide and dopamine quinones [44-46]. Therefore, enhanced expression of apoptosis-related genes in the PFC (as well as in the striatum) of cohort 2 SHRs is consistent with the observed neurotoxic effects of long-term psychostimulant exposure. Additionally, these findings may also reflect other previously described neurotoxic effects of chronic methylphenidate treatment such as decreased antioxidant defenses promoting peripheral oxidative adaptation [47], altered $\mathrm{Na}^{+}, \mathrm{K}^{+}$-ATPase activity in the cerebrum thereby affecting cellular excitability [48], downregulation of activity regulated cytoskeletal gene expression interfering with long-term potentiation and consolidation of long-term memory [49], as well as druginduced cognitive impairment on spatial reference and working memory tasks [50].

Of note, we observed a larger number of DEGs in the striatum when compared with the PFC in cohort 2 SHRs coinciding with the findings of our previous study [19], and corroborating the previous assumption on the crucial role of the striatum during compulsive drug use or abuse [51]. Interestingly, a majority of the DEGs in the striatum are downregulated genes associated with important cellular functions such as cell adhesion (e.g. Pcdh10, Ctbbd1, Itgb6), transcription (e.g. Notch3, Nsbp1, Sik1), mitochondrion organization (e.g. Prps18c, Letm1, Uqcrc2) and ubiquitinmediated proteolysis (e.g. Nedd4, Usp $27 x$, Ube2d2). The expression patterns of representative genes (e.g. Pcdh10, Ctnd1, Uqcrc2, Nedd4) from the different functional categories have been validated by confirmatory qRT-PCR 
analyses. However, it remains to be established whether changes in expression levels of these genes influenced methylphenidate reinforcement in drug-treated SHRs. Moreover, further studies are warranted to determine the contribution of these altered transcripts in the molecular mechanism of long-term recreational methylphenidate use or abuse in individuals with ADHD.

The decrease in Pcdh10 gene expression is both a novel and interesting observation considering the role of cell adhesion genes in maintaining neuronal and synaptic connections, and also in neuronal development [52]. Pcdh10, a member of the protocadherin gene family of cell adhesion molecules (CAMs), codes for a cadherin-related neuronal receptor involved in the establishment and function of specific cell-cell connections in the brain [53]. Furthermore, mutant analysis has demonstrated that loss of Pcdh10 can influence different aspects of development and post-natal life [48]. Whereas prior to this study there has been no experimental literature implicating the role of Pcdh10 in drug abuse, the involvement of this gene in a neurodevelopmental disorder, autism spectrum disorder, has already been reported [54]. Hence, downregulation of Pcdh10 gene expression observed in this study is an index of altered cell-to-cell communication, which may also signify disturbances in synaptic or neuronal plasticity within the striatal complex.

Altered gene expression of Uqcrc2 has also been confirmed by qRT-PCR. The Uqcrc2 gene is a component of the mitochondrial respiratory complex III, which codes for an enzyme involved in the electron transport chain. Furthermore, dysregulation of the expression of this gene has been implicated in some neuropsychiatric disorders such as bipolar disorder [55]. A previous study reported inhibition of mitochondrial respiratory chain complex III in the striatum as well as other brain regions of adult Wistar rats exposed to chronic (28 days) treatment of methylphenidate [56]. Downregulation of mitochondrial respiratory complex III (as well that of other complexes) has been viewed as a compensatory response in order to maintain energy homeostasis through enhancement of other metabolic systems [56]. Alternatively, it has been assumed to result from neurotoxic effects of methylphenidate via the generation of ROS [56]. Further studies are required to examine this point in detail. Nevertheless, the decrease in Uqcrc2 expression as observed in this study extends the previous findings [56], and indicates that methylphenidate treatment alters brain mitochondrial functions. These findings also suggest disruptions in brain energy metabolism in methylphenidate-treated and reinforced SHRs, although additional studies are required to confirm this.

Nedd4 gene expression has also been decreased in the striatum of cohort 2 SHR. Nedd4 belongs to a family of ubiquitin ligases, which has been shown to play key roles in both trafficking and degradation of proteins. In particular,
Nedd4 has been hypothesized to maintain target protein at the plasma membrane by balancing steady state insertion and retrieval of proteins critical to the development and neuronal function in the brain [57]. Although further studies are required, downregulation of Nedd4 in this study may indicate disruption in the removal of misfolded or unwanted proteins causing their accumulation. The pathological consequences of this altered process in the context of long-term methylphenidate abuse are still unknown, although accumulation of unnecessary proteins resulting from a defective ubiquitin dependent proteolysis has been shown to contribute to aggregation events, a pathogenic mechanism in several neurobehavioral and neurodegenerative disorders [58,59]. Furthermore, in view of the important roles of ubiquitin system in synaptic growth and function, downregulation of Nedd4 may also affect synaptic growth and plasticity, contributing to altered brain function.

\section{Conclusion}

Altered expression of genes associated with apoptosis, cell adhesion, mitochondria organization, ubiquitinmediated proteolysis, etc. in the PFC and/or striatum of methylphenidate-treated SHRs which showed methylphenidate CPP and self-administration indicate methylphenidate-induced neurotoxicity, alterations in synaptic and neuronal plasticity, energy metabolism and ubiquitin-dependent protein degradation. In addition, these changes may also reflect cognitive impairment associated with chronic methylphenidate use as demonstrated in preclinical studies [e.g. [49,50]]. Future studies should be performed to determine the link between changes in the expression of these genes and influence on methylphenidate reinforcement in SHRs. Moreover, additional studies are warranted to determine the clinical significance of the findings of this study with regard to methylphenidate abuse or addiction in individuals with ADHD. Nevertheless, this study presents new research directions and interesting topics for further investigation. For instance, it would be worthwhile to examine molecular changes that occur in the brains of methylphenidatepretreated and methylphenidate-reinforced WKY. Providing answers to this query will establish whether the reported gene expression changes are exclusive to SHR and not possibly due to inbreeding. Furthermore, investigating gene expression changes in methylphenidate-pretreated animals which showed less methylphenidate CPP and selfadministration will also provide important information not only on the mechanisms of methylphenidate abuse in ADHD individuals, but also on protection against methylphenidate addiction via development of novel targets for the treatment of methylphenidate or psychostimulant addiction. Along these lines, complementary findings can be obtained by comparing gene expression patterns 
associated with adolescent exposure to methylphenidate and atomoxetine, another widely-used ADHD drug without stimulant-like effects and assumed to be devoid of abuse liability, in view results from a previous study which showed differential effects of these drugs in SHRs [60].

\section{Additional files}

Additional 1: Table S1A. Upregulated genes in the striatum. Table S1B. Downregulated genes in the striatum.

Additional 2: Table S2. Differentially expressed genes in the striatum of cohort 2 SHRs relative to control.

\section{Competing interests}

The authors declare that they have no competing interests.

\section{Authors' contributions}

ID, JHC and MSN conceived and designed the study. ID, HJK and ARS performed the SA and CPP experiments. ID, MSN and JHC analyzed the microarray data and ID, ARS performed the statistical analysis. JHR, CYS, JHC, $\mathrm{BNK}$ and $\mathrm{DHH}$ provided insights on interpreting the results. All authors participated in drafting and in writing the final revision of the manuscript. All authors read and approved the final manuscript.

\section{Acknowledgements}

This research was supported by funds from the National Research Foundation of Korea (NRF) and the Korea Healthcare Technology Research and Development Project, Ministry for Health and Welfare Affairs, Korea (grant number A120013).

\section{Author details}

${ }^{1}$ Uimyung Research Institute for Neuroscience, Sahmyook University, 26-21 Kongreung-2-dong, Hwarangro- 815 Nowon-gu, Seoul 139-742, Korea. ${ }^{2}$ Division of Child and Adolescent Psychiatry, Clinical Research Institute, Seoul National University Hospital, 28 Yungundong, Chongrogu, Seoul 110-744, Korea. ${ }^{3}$ Department of Psychiatry, Chung-Ang University Medical School, 102 Heukseok-ro, Dongjak-gu, Seoul 156-755, Korea. ${ }^{4}$ Department of Oriental Pharmaceutical Science, Kyung Hee East-west Pharmaceutical Research Institute, College of Pharmacy, Kyung Hee University, Seoul 472-864, Korea. ${ }^{5}$ Department of Neuroscience, School of Medicine, Konkuk University, Seoul 143-701, Korea. ${ }^{6}$ Natural Products Research Institute, College of Pharmacy, Seoul National University, 1 Gwanak-ro, Gwanak-gu, Seoul 151-742, Korea.

Received: 22 January 2014 Accepted: 25 April 2014

Published: 6 May 2014

\section{References}

1. Heal DJ, Cheetham SC, Smith SL: The neuropharmacology of ADHD drugs in vivo: insights on efficacy and safety. Neuropharmacology 2009, 57:608-618,

2. Solanto MV: Neuropsychopharmacological mechanisms of stimulant drug addiction in attention-deficit hyperactivity disorder: a review and integration. Behav Brain Res 1998, 94:127-152.

3. Kiyatkin EA, Rebec GV: Dopaminergic modulation of glutamate-induced excitations of neurons in the neostriatum and nucleus accumbens of awake, unrestrained rats. J Neurophysiol 1996, 75:142-153.

4. Volkow ND, Swanson JM: Variables that affect the clinical use and abuse of methylphenidate in the treatment of ADHD. Am J Psychiatry 2003, 160:1909-1918.

5. Volkow ND, Wang GJ, Fowler JS, Logan J, Gatley J, Wong C, Hitzemann R, Pappas NR: Reinforcing effects of psychostimulants in humans are associated with increases in brain dopamine and occupancy of D2 receptors. J Pharmacol Exp Ther 1999, 291:409-415.

6. Dalley JW, Theobald D, Cerry JA, Milstein K, Laane BJ, Everitt TW: Cognitive sequelae of intravenous amphetamine self-administration in rats: evidence for selective effects on attentioanal performance. Neuropsychopharmacology 2005, 30:525-537.

7. Fletcher PJ, Korth KM, Stewart J, Chambers JW: Depletion of brain serotonin following intra-raphe injections of 5,7-dihydroxytryptamine does not alter d-amphetamine self-administration across different schedules and access conditions. Psychopharmacology (Berl) 1999, 146:185-193.

8. Marco EM, Adriani W, Ruocco LA, Canese R, Sadile AG, Laviola G: Neurobehavioral adaptations to methylphenidate: The issue of early adolescent exposure. Neurosci Biobehav Rev 2011, 35:1722-1739.

9. Augustyniak PN, Kourrich S, Rezazadeh SM, Stewart J, Arvanitogiannis A: Differential behavioral and neurochemical effects of cocaine after early exposure to methylphenidate in an animal model of attention deficit hyperactivity disorder. Behav Brain Res 2006, 167:379-382.

10. Brandon $C L$, Marinelli M, White FJ: Adolescent exposure to methylphenidate alters the activity of rat midbrain dopamine neurons. Biol Psychiatry 2003, 54:1338-1344.

11. Harvey RC, Sen S, Deaciuc A, Dwoskin LP, Kantak KM: Methylphenidate treatment in adolescent rats with an attention deficit/hyperactivity disorder phenotype: cocaine addiction vulnerability and dopamine transporter function. Neuropsychopharmacology 2011, 36:837-847.

12. Kollins SH: ADHD, substance use disorders, and psychostimulant treatment: current literature and treatment guidelines. J Atten Disord 2008, 12(2):115-125

13. Soeters HS, Howells FM, Russell VA: Methylphenidate does not increase ethanol consumption in a rat model for attention-deficit hyperactivity disorder-the spontaneously hypertensive rat. Metab Brain Dis 2008, 23:303-314.

14. Steiner $H$, Van Waes $V$ : Addiction-related gene regulation: risks of exposure to cognitive enhancers vs. other psychostimulants. Prog Neurobiol 2013, 100:60-80.

15. Yuferov V, Nielsen D, Butelman E, Kreek MJ: Microarray studies of psychostimulant-induced changes in gene expression. Addict Biol 2005, 10:101-118.

16. Rhodes JS, Crabbe JC: Gene expression induced by drugs of abuse. Curr Opin Pharmacol 2005, 5(1):26-33.

17. Freeman WM, Lull ME, Patel KM, Brucklacher RM, Morgan D, Roberts D, Vrana KE: Gene expression changes in the medial prefrontal cortex and nucleus accumbens following abstinence from cocaine self-administration. BMC Neurosci 2010, 11:29.

18. Robison AJ, Nestler EJ: Transcriptional and epigenetic mechanisms of addiction. Nat Rev 2011, 12:623-637.

19. Dela Peña IC, Jeon SJ, Lee E, Ryu JH, Shin CY, Noh M, Cheong JH: Neuronal development genes are key elements mediating the reinforcing effects of methamphetamine, amphetamine, and methylphenidate. Psychopharmacology 2013, 230:399-413.

20. Fan X, Bruno KJ, Hess EJ: Rodent models of ADHD. Curr Top Behav Neurosci 2012, 9:273-300.

21. Sagvolden T, Russell VA, Aase H, Johansen EB, Farshbaf M: Rodent models of attention-deficit/hyperactivity disorder. Biol Psychiatry 2005, 57:1239-1247.

22. Russell VA, Sagvolden T, Johansen EB: Animal models of attention-deficit hyperactivity disorder. Behav Brain Funct 2005, 1:9.

23. Sagvolden T: Behavioral validation of the spontaneously hypertensive rat (SHR) as an animal model of attention-deficit/hyperactivity disorder (AD/HD). Neurosci Behav Rev 2000, 24:31-39.

24. Vendruscolo LF, Izidio GS, Takahashi RN: Drug reinforcement in a rat model of attention deficit/hyperactivity disorder. Curr Drug Abuse Rev 2009, 2(2):177-183.

25. Harlan R, Garcia M: Drugs of abuse and immediate-early genes in the forebrain. Mol Neurobiol 1998, 16:221-267.

26. Berke JD, Hyman SE: Addiction, dopamine and the molecular mechanisms of memory. Neuron 2000, 25:515-532.

27. Gerdeman GL, Partridge JG, Lupica CR, Lovinger DM: It could be habit forming: drugs of abuse and striatal synaptic plasticity. Trends Neurosci 2003, 26:184-192.

28. Kalivas PW, Volkow ND: New medications for drug addiction hiding in glutamatergic neuroplasticity. Mol Psychiatry 2011, 16:974-986.

29. Qiu J, Hong Q, Chen RH, Tong ML, Zhang M, Fei L, Pan XQ, Guo M, Guo XR, Chi X: Gene expression profiles in the prefrontal cortex of SHR rats by cDNA microarrays. Mol Biol Rep 2010, 37:1733-1740.

30. Dela Peña IC, Lee JC, Lee HL, Woo TS, Lee HC, Sohn AR, Cheong JH: Differential behavioral responses of the spontaneously hypertensive rat to methylphenidate and methamphetamine: lack of a rewarding effect of repeated methylphenidate treatment. Neurosci Lett 2012, 514:189-193.

31. Kollins SH: A qualitative review of issues arising in the use of psycho-stimulant medications in patients with ADHD and co-morbid substance use disorders. Curr Med Res Opin 2008, 24:1345-1357. 
32. Mannuzza S, Klein RG, Truong NL, Moulton JL III, Roizen ER, Howell KH: Age of methylphenidate treatment initiation in children with ADHD and later substance abuse: prospective follow-up into adulthood. Am J Psychiatry 2008, 165:604-609.

33. Gerasimov MR, Franceschi M, Volkow ND, Gifford A, Gatley SJ, Marsteller D, Molina PE, Dewey SL: Comparison between intraperitoneal and oral methylphenidate administration: microdialysis and locomotor activity study. J Pharmacol Exp Ther 2000, 295:51-57.

34. Dela Peña IC, Ahn HS, Choi JY, Shin CY, Ryu JH, Cheong JH: Methylphenidate self-administration and conditioned place preference in an animal model of attention-deficit hyperactivity disorder: the spontaneously hypertensive rat. Behav Pharmacol 2011, 22:31-39. doi:10.1097.

35. Huang DW, Sherman BT, Lempicki RA: Systematic and integrative analysis of large gene lists using DAVID bioinformatics resources. Nat Protocols 2008, 4:44-57.

36. Livak KJ, Schmittgen TD: Analysis of relative gene expression data using real-time quantitative PCR and the 2(-Delta Delta $C(T)$ ) method. Methods 2001, 25:402-408.

37. Robinson TE, Berridge KC: The neural basis of drug craving: an incentivesensitization theory of addiction. Brain Res Rev 1996, 18:247-291.

38. Dela Peña IC, Cheong $\mathrm{JH}$ : Abuse and dependence liability assessment of methylphenidate in the spontaneously hypertensive rat model of attention-deficit/hyperactivity disorder (ADHD): what have we learned? Arch Pharm Res 2013, 23:400-410.

39. Biederman JJ, Wilens T, Mick E, Faraone SV, Weber W, Curtis S, Thornell A, Pfister $\mathrm{K}$, Jetton J, Soriano J: Is ADHD a risk factor for psychoactive substance use disorders? Findings from a four-year prospective follow-up study. J Am Acad Child Adolesc Psychiatr 1997, 36:21-29.

40. Yui S, Nakatani Y, Mikami M: Calprotectin (S100a8/S100a9), an inflammatory protein complex from neurophils with a broad apoptosis-inducing activity. Biol Pharm Bull 2003, 26:753-760.

41. Sadasivan S, Pond BB, Pani AK, Qu C, Jiao Y, Smeyne RJ: Methylphenidate exposure induces dopamine neuron loss and activation of microglia in the basal ganglia of mice. PloS One 2012, 7:e33693.

42. Baldwin HA, Colado MI, Murray TK, De Souza RJ, Green AR: Striatal dopamine release in vivo following neurotoxic doses of methamphetamine and effect of the neuroprotective drugs, chlormethiazole and dizolcilpine. Brit J Pharmacol 1993, 108:590-596.

43. Clark KH, Wiley CA, Bradberry CW: Psychostimulant abuse and neuroinflammation: emerging evidence of their interconnection. Neurotox Res 2012, 23:174-188.

44. Filloux F, Townsend JJ: Pre- and postsynaptic neurotoxic effects of dopamine demonstrated by intrastriatal injection. Exp Neurol 1993, 119:79-88.

45. Hastings TG, Lewis DA, Zigmond MJ: Role of oxidation in the neurotoxic effects of instrastriatal dopamine injections. Proc Natl Acad Sci U S A 1996, 93:1956-1961.

46. Gomes KM, Inacio CG, Valvassori SS, Reus GZ, Boeck CR, Dal-Pizzol F, Quevedo J: Superoxide production after acute and chronic treatment with methylphenidate in young and adult rats. Neurosci Lett 2009 465(1):95-98

47. Schmitz F, Scherer EB, da Cunha MJ, da Cunha AA, Lima DD, Delwing D, Netto CA, Wyse AT: Chronic methylphenidate administration alteres antioxidant defenses and butyrylcholinesterase activity in blood of juvenile rats. Mol Cell Biochem 2012, 361:281-288.

48. Chase T, Carrey N, Soo E, Wilkinson M: Methylphenidate regulates activity regulated cytoskeletal associated but not brain-derived neurotrophic factor gene expression in the developing rat striatum. Neuroscience 2007, 144:969-984.

49. Scherer EB, Matte C, Ferreira AG, Gomes KM, Comim CM, Mattos C, Quevedo J, Streck EL, Wyse AT: Methylphenidate treatment increases $\mathrm{Na}^{+}, \mathrm{K}^{+}$-ATPase activity in the cerebrum of young and adult rats. J Neural Transm 2009, 116:1681-1687.

50. Scherer EB, da Cunha MJ, Matte C, Schmitz F, Netto C, Wyse AT: Methylphenidate affects memory, brain-derived neurotrophic factor immunocontent and brain acetylcholinesterase activity in the rat Neurobiol Learn Mem 2010, 94(2):247-253.

51. Koob GF, Volkow ND: Neurocircuitry of addiction. Neuropsychopharmacology 2010, 35:217-238.
52. Uemura M, Nakao S, Suzuki ST, Takeichi M, Hirano S: OL-protocadherin is essential for growth of striatal axons and thalamocortical projections. Nat Neurosci 2007, 10:1151-1159.

53. Yagi T: Clustered protocadhrin family. Dev Growth Differ 2008, 50(1):S131-S140.

54. Morrow EM, Yoo SY, Flavell SW, Kim TK, Lin Y, Hill RS, Mukaddes NM, Balkhy S, Gascon G, Hashmi A, Al-Saad S, Ware J, Joseph RM, Greenblatt R, Gleason D, Ertelt JA, Apse KA, Bodell A, Partlow JN, Barry B, Yao H, Markianos K, Ferland $\mathrm{R}$, Greenberg ME, Walsch CA: Identifying autism loci and genes by tracing recent shared ancestry. Science 2008, 321:218-223.

55. Sun X, Wang JF, Tseng M, Young LT: Downregulation in components of the mitochondrial electron transport chain in the postmortem frontal cortex of subjects with bipolar disorder. J Psychiatry Neurosci 2006, 31:189-196.

56. Fagundes AO, Scaini G, Santos PM, Sachet MU, Bernhardt NM, Rezin GT, Valvassori SS, Schuck PF, Quevedo J, Streck EL: Inhibition of mitochondrial respiratory chain in the brain of adult rats after acute and chronic administration of methylphenidate. Neurochem Res 2010, 35:405-411.

57. Donovan P, Poronnik P: Nedd4 and Nedd4-2: ubiquitin ligases at work in the neuron. Int J Biochem Cell Biol 2013, 45(3):706-710.

58. Lam YA, Pickart CM, Alban A, Landon M, Jamieson C, Ramage R, Mayer RJ, Layfield R: Inhibition of the ubiquitin-proteasome system in Alzheimer's disease. Proc Natl Acad Sci 2000, 97:9902-9906.

59. Layfield R, Alban A, Mayer RJ, Lowe J: The ubiquitin protein catabolic disorders. Neuropathol Appl Neurobiol 2001, 27:171-179.

60. Fumagalli F, Cattaneo A, Caffino L, Ibba M, Racagni G, Carboni E, Gennarell M, Riva MA: Sub-chronic exposure to atomoxetine up-regulates BDNF expression and signallin in the brain of adolescent spontaneously hypertensive rats: comparison with methylphenidate. Pharmacol Res 2010, 62(6):523-529.

doi:10.1186/1744-9081-10-17

Cite this article as: dela Peña et al:: Prefrontal cortical and striatal transcriptional responses to the reinforcing effect of repeated methylphenidate treatment in the spontaneously hypertensive rat, animal model of attention-deficit/hyperactivity disorder (ADHD). Behavioral and Brain Functions 2014 10:17

\section{Submit your next manuscript to BioMed Central and take full advantage of:}

- Convenient online submission

- Thorough peer review

- No space constraints or color figure charges

- Immediate publication on acceptance

- Inclusion in PubMed, CAS, Scopus and Google Scholar

- Research which is freely available for redistribution

Submit your manuscript at www.biomedcentral.com/submit
C Biomed Central 